\title{
Multi-belt Onion-ring Visualization of OF@TEIN Testbed for SmartX Multi-View Visibility \\ Muhammad Usman ${ }^{1}$, Nguyen Tien Manh ${ }^{2}$ and JongWon Kim ${ }^{1+}$ \\ ${ }^{1}$ Gwangju Institute of Science \& Technology, South Korea \\ ${ }^{2}$ Hanoi University of Science \& Technology, Vietnam
}

\begin{abstract}
ICT infrastructure visualization based on collected visibility data is essential for the effective operation of contemporary SDN (Software-Defined Networking)-enabled cloud playground. More precisely, collecting and visualizing visibility information about various physical, virtual, and container $(p+v+c)$ types of resources is one of the key requirement for multisite cloud playground operators. In order to address the playground visibility challenges, we have been developing a multi-layered visibility and visualization solution denoted as SmartX Multi-View Visibility Framework (MVF). By extending our earlier effort, in this paper, we attempt to realize multi-belt onion-ring style visualization software to assist both playground developers and operators in capturing the end-to-end visualization of infrastructure resources and flows.
\end{abstract}

Keywords: Multisite cloud, software-defined networks, multi-layer visibility, onion-ring visualization.

\section{Introduction}

Unlike traditional network-focused testbeds, Future Internet testbeds should provide experimental networking facility without limiting the number of concurrent users with different resource requirements, the number or type of supported services, and most importantly the topological shape of deployed networks. Thanks to the emerging SDN (Software-Defined Networking) paradigm, open and programmable types of experimental networks are beginning to be deployed in the construction and operation of multisite testbeds. Aligned with Future Internet testbed projects like GENI [1] and FIRE [2], we launched OF@ TEIN project [3] over TEIN (Trans-Eurasia Information Network) in 2012, which is now expanded to an SDN-enabled multisite clouds [4]. As shown in Fig. 1, OF@TEIN multisite cloud (denoted as OF@TEIN Playground) connects around 10 international sites spread across nine member countries of TEIN project.

OF@TEIN Playground consists of multiple types of resources: physical, virtual, and container types. Generally, physical servers and switches, and physical interconnects are referred as physical resources. Virtual machine instances, virtual switches, and virtual interconnects are referred as virtual resources. Container instances recently entered the scene to provide a new light-weight category of resource for flexible workload deployment. In order to effectively operate OF@TEIN Playground, it is truly imperative to recognize where physical, virtual, and container resources are located and how they are inter-related to each other. Therefore, in this paper, we propose multi-belt onion-ring style visualization, as a helper solution to the above challenge of single view unified visualization. To effectively realize onion-ring visualization, diverse types and volume of visibility data are required to be collected, processed, and interpreted. However, to the best of our knowledge, existing open-source solutions does not provide the required onion-ring visualization capability to directly match the multisite resource infrastructure of OF@TEIN Playground.

Lately we have been developing an integrated framework for multi-layered visibility workflow, denoted as 'SmartX Multi-View Visibility Framework (MVF)' [5]. By applying SmartX MVF to OF@TEIN

\footnotetext{
${ }^{+}$Corresponding author. Tel.: + (82-62-715-2219); fax: +(82-62-715-3155).

E-mail address:(jongwon@gist.ac.kr).
} 
Playground, we are able to monitor the various dynamic visibility metrics from various measurement points across both physical and virtualized resources and associated flows of the playground. Thus, in this paper, we first leverage the already collected playground visibility data with slight modifications followed by careful integration (i.e., organized and interpreted) to enable the required onion-ring visualization. In summary, the key contributions of this paper are the design, prototype implementation, and verification of multi-belt onion-ring visualization for OF@ TEIN Playground.

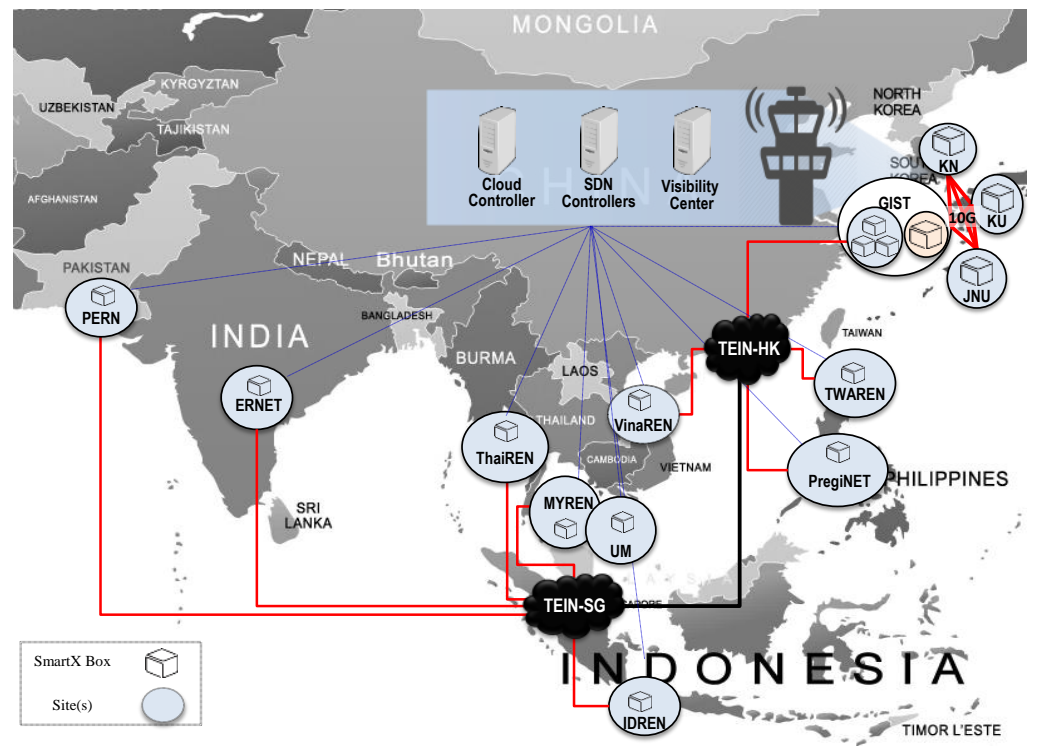

Fig. 1: OF@TEIN Playground as multisite clouds.

The remainder of this paper is organized as follows. In Section II, we briefly discuss SmartX MVF and issues with its current visualization support. In Section III, we collectively cover both design and preliminary prototype implementation of onion-ring visualization. After that, we discuss verification results for proposed onion-ring visualization in Section IV. Finally, Section V concludes the paper.

\section{Background and Related Work}

\subsection{SmartX Multi-View Visibility Framework}

SmartX MVF is proposed to deal with the multiple layers of visibility and visualization of OF@ TEIN Playground [5]. That is visibility framework is mainly designed to deal with four layers such as resourcelayer, slice-layer, flow-layer, and workload-layer. Resource-layer visibility is responsible for monitoring and visualization of playground physical resources and inter-connects (e.g. paths and links). Slice-layer visibility covers monitoring and visualization of isolated virtualized resources and their virtual interconnects that are configured on a physical resource. Flow-layer visibility is responsible for providing different levels of flowcentric information by utilizing flow collection, clustering, and tagging schemes to assist SDN-based flexible networking (i.e. steering/ mapping). Lastly, workload-layer visibility is responsible for the monitoring and visualization of inter-connected functions and their placements for tenant-based applications. Also, for visibility data processing, SmartX MVF has four main stages. Visibility Collection and Validation stage collects and validates data based on selected visibility metrics. Cleaned data is sent to Visibility Storage and Staging stage that is responsible for data storage. After that, Visibility Integration stage fuses collected data for identifying key patterns and identifies any anomalies. Finally, Visualization stage accesses processed data and transforms it into graphical views.

\subsection{Limitation of Existing Tools}

A number of open-source visibility monitoring and visualization tools are already available; but, they do not directly match unique single-box-virtualized deployment style of OF@TEIN Playground. In order to solve this shortcoming, we have developed $\mathrm{p}+\mathrm{v}$ topological visualization for SmartX Multi-V iew Visibility [6]. This $\mathrm{p}+\mathrm{v}$ topological visualization is in a tree-style view, focusing on the interconnections among distributed physical and virtual resources. However, this flat tree-style topological visualization is proving 
insufficient to effectively demonstrate multi-layer visibility of multisite resource infrastructure of OF@TEIN Playground. Therefore, a more systematic and fine-grained visualization with clear separation between multiple layers and multiple sites is essential for SmartX Multi-View Visibility to effectively operate OF@TEIN Playground.

\subsection{Requirements of Multi-Belt Onion-Ring Visualization}

Multi-belt onion-ring visualization should meet the following requirements in order to fully qualify as a capable solution for OF@TEIN Playground. Firstly, onion-ring visualization should be innovative to offer a single unified view for SDN-enabled multisite clouds by covering various $\mathrm{p}+\mathrm{v}+\mathrm{c}$ resource components and their associated flows of OF@TEIN Playground. Also, for faster troubleshooting, this onion-ring visualization must define several dedicated belts to flexibly incorporate multi-layer visibility data that is collected from multiple sites. Furthermore, multi-tenancy support is another key requirement that onion-ring visualization should support.

\section{Multi-Belt Onion-Ring Visualization: Design and Prototype Implementation}

\subsection{Selected Tools for Playground Visibility Measurements}

Initially, the operation of visibility collection requires the identification of key performance metrics that affirm major impact on the operational states of the playground. Currently, for resource-layer visibility collection, we extensively utilize Intel Snap monitoring framework [7]. Besides, for flow-layer visibility collection, we use sFlow-based sampling and eBPF-based tracing tools [8]. In addition, to diversify the visibility collection of resource, slice, and flow layers, we collect visibility metrics from both cloud and SDN controller(s) as well

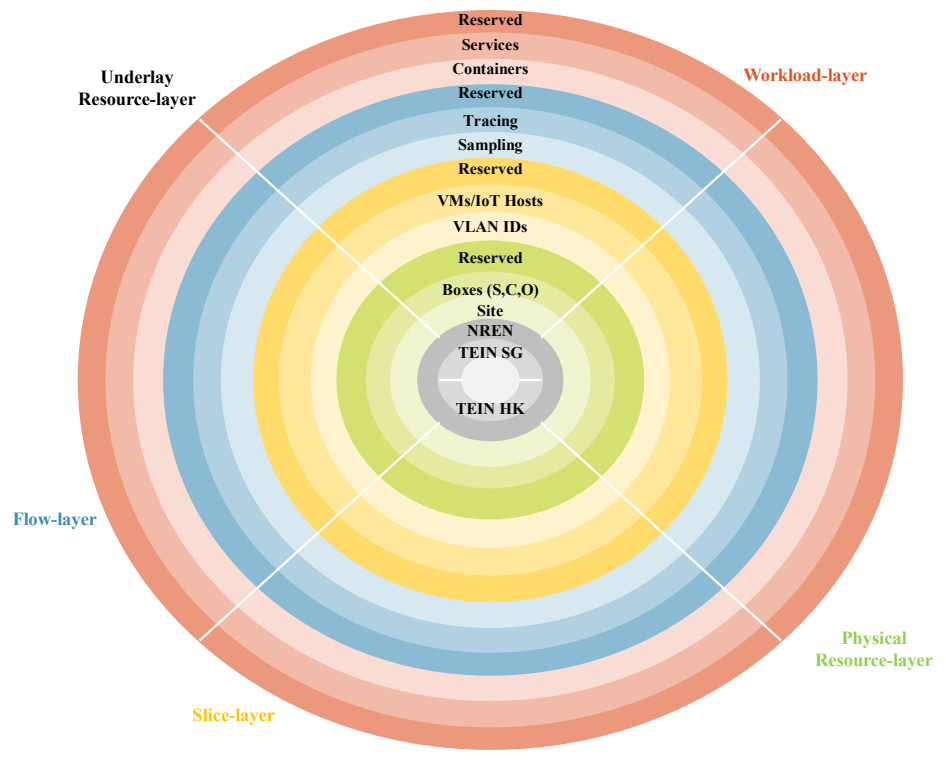

Fig. 2: Design of onion-ring visualization for SmartX Multi-View Visibility.

\subsection{Design and Prototype Implementation}

To address the above highlighted difficulties of playground visualization, we focus on multi-belt onionring style visualization as shown in Fig. 2. Tiered belts on the surface partly cover the multiple layer visualization requirements of SmartX Multi-View Visibility. Primarily there are five main belts for each layer of visibility and then they are further divided into three sub-belts to allow incorporation of additional information for display in a particular belt. Also, the color-coded areas that are separated with white lines correspond to distributed sites that host physical, virtualized, or containerized resources (e.g., boxes, switches, and service functions).

In order to realize the multi-belt onion-ring visualization, we leverage open-source visualization library called psd3 [9]. That is, psd3 is primarily based on D3.js and supports multi-level pie charts. To easily visualize performance metrics data and observe various trends, we utilize open-source software named 
Grafana. Finally, for onion-ring visualization deployment, Node.js JavaScript runtime is selected due to its non-blocking I/O and event-driven features.

\subsection{Visibility Data Preparation for Multi-Belt Onion-Ring Visualization}

The collected multi-layer visibility data is stored into so-called Visibility DataLake [5]. Currently, we extensively utilize MongoDB to store playground configuration and various playground entities status data while InfluxDB and Elasticsearch are used to store near-real-time metrics data and flows data respectively. Please note that MongoDB (document-oriented), Elasticsearch (index-oriented), and InfluxDB (time series) are all special-purpose NoSQL DataStores. More specifically, to enable onion-ring visualization, we rely on separate database that stores updated configuration and status data for the different entities of OF@TEIN Playground. This configuration or status database is managed via MongoDB collections. For example, each layer of the onion-ring has an associated and dedicated collection. Then to facilitate data integration across different collections, we define specific keys (e.g., physical box identifier). At the backend, Java-based plugins are used to update playground entities states. That is, Java-based plugins are explic itly developed for collecting and updating data in MongoDB collections on regular time intervals.

\section{Multi-Belt Onion-ring Visualization: Verification}

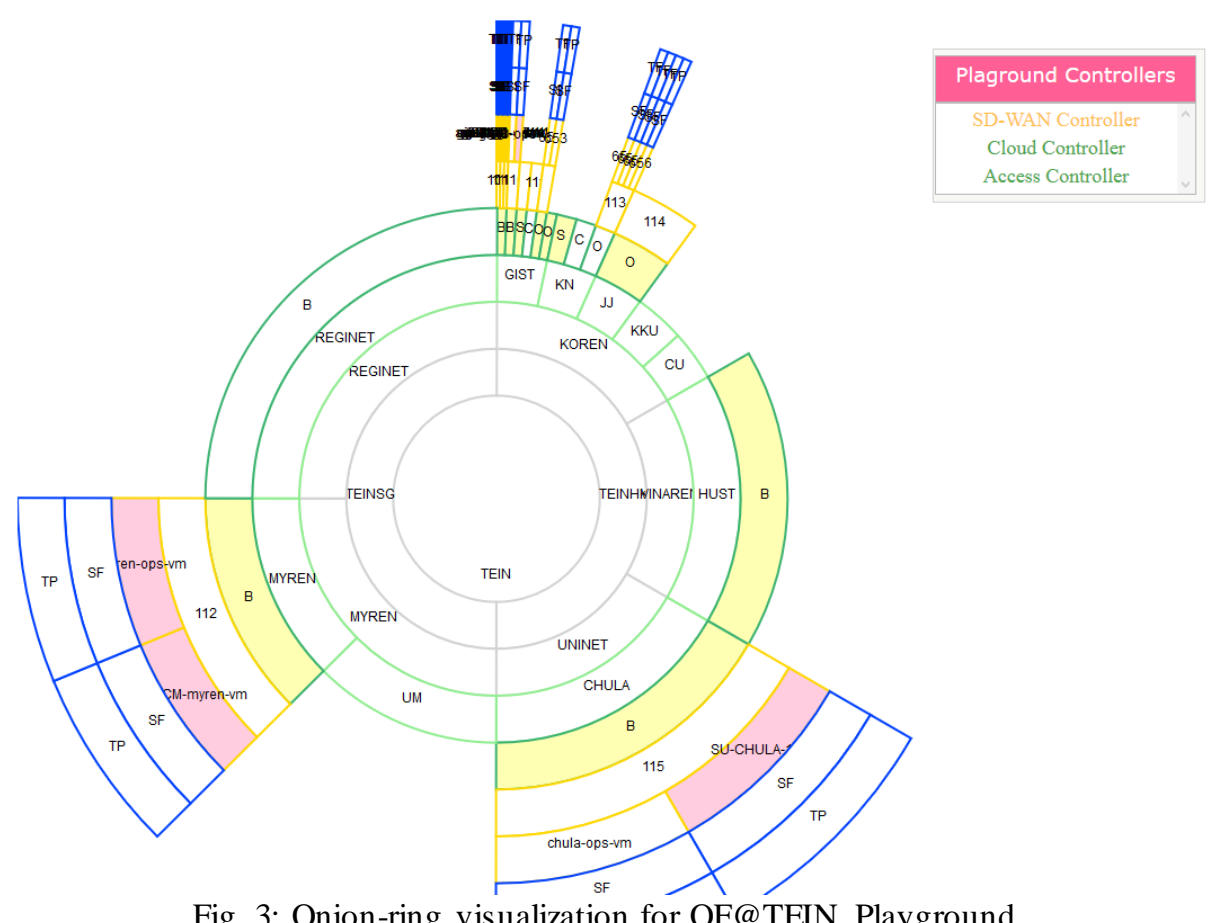

Fig. 3: Onion-ring visualization for OF@TEIN Playground.

For verifying the prototype implementation, we utilize OF@TEIN testbed [4]. We consider a simple configuration, for instance virtual boxes are deployed over physical boxes and virtual boxes are interconnected through VXLAN-based L2 overlay network. Fig. 3 shows a snapshot of multi-belt onion-ring visualization. This dashboard is effective in presenting underlay resource-layer, physical resource-layer, slice-layer, and flow-layer visibilities together in a single unified view. At the underlay resource layer, that is from the center to outside, we can see international network PoPs (TEINSG and TEINHK), national PoPs (e.g., MYREN, KOREN, ...) and sites (e.g., GIST, CHULA, ...). On top of the distributed sites, physical boxes are shown with their respective type $(\mathrm{B}, \mathrm{C}, \mathrm{S})$. Next belt shows the VLAN IDs configured for particular tenants on a physical box. Few physical boxes also have a number of virtual boxes (VMs) within them, respectively. For example, type B physical box in MYREN site have two virtual boxes and type O box in KN site have 4 virtual boxes. Next two belts in blue color show sampled flows and traced packets of each individual virtual box. Please note that, with the help of associated box IDs, we can easily visualize resource usage graphs in the boxes. Thus, with the support of visualization dashboard, the operators and developers of playground can easily grasp the overlay-based playground topology and check its relationship with the 
underlay WAN networks that belong to separate network operators. Also, on the top right corner, we can see the list of playground controllers with their online working status.

\section{Conclusion and Future Work}

In this paper, we presented our initial effort to provide the multi-belt onion-ring visualization for OF@TEIN Playground developers and operators to effectively visualize multiple layers of visibilities together. However, the proposed onion-ring visualization still needs further refinements to reach the eventual goal of visualizing multi-view visibility. Thus, as a second step toward our goal, we are currently working to incorporate visualization of workload-layer visibility into onion-ring.

\section{Acknowledgement}

This work was supported in part by Institute for Information \& communications Technology Promotion (IITP) grant funded by the Korea government (MSIT) (No. 2015-0-00575, Global SDN/NFV Open-Source Software Core Module/Function Development). This work was also supported in part by Institute for Information \& communications Technology Promotion (IITP) grant funded by the Korea government (MSIT) (No. 2017-0-00421, Cyber Security Defence Cycle Mechanism for New Security Threats).

\section{References}

[1] M. Berman et al. GENI: A federated testbed for innovative network experiments. Computer Networks, 2014, vol. 61 , pp. 5-23.

[2] FIRE: Future Internet Research and Experimentation, https://www.ict-fire.eu/.

[3] A. C. Risdianto and J. Kim. Prototyping media distribution experiments over OF@TEIN SDN-enabled testbed. In: Proc. of APAN-NRW, Nantou, 2014, pp. 12-18.

[4] A. C. Risdianto, P. W. Tsai, C. Yang, T. C. Ling, and J. Kim. Leveraging open-source software for federated multisite SDN-Cloud playground. In: Proc. of 2016 IEEE NetSoft Conference and Workshops (NetSoft), Seoul, 2016, pp. 423-427.

[5] M. Usman, A. C. Risdianto, J. Han, M. Kang, and J. Kim. SmartX MultiView visibility framework leveraging open-source software for SDN-Cloud playground. In: Proc. of IEEE NetSoft Conference and Workshops (NetSoft), Bologna, 2017, pp. 1-4.

[6] M. Usman, A. C. Risdianto, J. Han, J. Kim, and Nguyen Van Huynh. Physical-virtual topological visualization of OF@TEIN SDN-enabled multi-site cloud. In: Proc. of International Conference on Information Networking (ICOIN), Da Nang, 2017, pp. 622-624.

[7] Intel Snap Telemetry Framework, http://snap-telemetry.io/.

[8] T. Nam and J. Kim. Prototype Implementation of Site Visibility Framework employing IO Visor-based Packet Tracing. In: Proc. The 12th KIPS International Conference on Ubiquitous Information Technologies and Applications (CUTE 2017), Taichung, 2017, pp. 1295-3000.

[9] Psd3, http://pshivale.github.io/psd3/. 